Thorax (1970), 25, 190.

\title{
Giant left atrium
}

\author{
B. T. LE ROUX and M. S. GOTSMA N
}

The Thoracic Surgical and Cardiac Units of the University of Natal, Wentworth Hospital, Durban

\begin{abstract}
Radiographic evidence of asymmetrical enlargement of the left atrium without atrial infarction is presented. Giant atrial enlargement is rarely symmetrical, the atrial appendage can contribute to the enlargement, and the giant atrium can be effectively trimmed. This will reduce its size and, therefore, the space which it occupies, and will restore the anatomical relations of neighbouring structures such as the main bronchi.
\end{abstract}

Enlargement of the left atrium is a feature of mitral valve disease, whether the valve is stenotic, incompetent, or both. The left atrium may achieve proportions which have provoked use of descriptive terms such as 'giant', 'massive', or 'aneurysmal'-most often when the mitral valve has been grossly incompetent (Parsonnet, Bernstein, and Martland, 1946), but also with a predominantly or exclusively stenotic valve (Venner, 1954 ; Kent, Fisher, Ford, and Neville, 1956). Apart from the haemodynamic consequences of a greatly enlarged left atrial chamber, the very size of the chamber constitutes an important disadvantage, with compression and displacement of neighbouring structures. These purely anatomical considerations were the subject of most of the earlier descriptions of the lesion, including probably the earliest-by Owen and Fenton (1901). Reference to these earlier descriptions of the lesion are contained in the paper by Parsonnet et al. (1946). Daley and Franks (1949) described the clinical features and anatomical consequences-dysphagia from oesophageal displacement, with the oesophagus either grossly stretched and flattened, or compressed against the vertebrae, if it has not slipped to one or other side ; splaying of the main bronchi and bronchial compression, sometimes sufficient to obstruct completely a lobar bronchus, especially the left lower and the middle lobar bronchi ; interruption of the left recurrent laryngeal nerve ; and occasionally even erosion of the spine. Oesophageal compression against the vertebrae by massive left atrial enlargement may not only cause dysphagia but also oesophageal erosion with death from haemorrhage (Le Roux and Williams, 1969).

It has been maintained that a giant left atrium exhibits symmetrical enlargement which is not more than an exaggeration of the usual symmetri- cal enlargement of the left atrium so common in mitral stenosis; that it is unusual for the atrial appendage to share in the generally gross enlargement; and that localized enlargements do not occur unless there is an associated atrial infarct (Daley and Franks, 1949). The purpose of this paper is to illustrate that truly giant left atrial enlargement is not, and in fact cannot be, symmetrical, that the atrial appendage may enlarge massively along with the body of the left atrium, and that amputation of a large part of a giant left atrium contributes to rapid recovery when obstruction at the mitral valve has been surgically corrected.

The normal left atrium comprises much of the posterior surface of the heart. The four pulmonary veins enter the left atrium at the corners of a square, the sides of which are rarely longer than $4 \mathrm{~cm}$. Anterior to the entrance of the right pulmonary veins there is a width of left atrial wall seldom, unless surgically developed, greater than $1 \mathrm{~cm}$. posterior or postero-lateral to the atrial septum and before the right atrium begins to form the right heart border. The left pulmonary veins have, anterior to their entrance into the left atrial chamber, an extent of left atrial wall greater than the right veins but normally not more than 2 or $3 \mathrm{~cm}$., and the left atrial appendage rides high on this aspect of the left atrium. Rostral to the orifices of the upper veins and caudal to those of the lower veins the extent of atrial wall is variable but again small; the immediate posterior relation of the atrial wall between the veins is the oblique sinus. Standard anatomical illustrations depict the left atrium at the level of the transverse sinus (Fig. 1), where a cross-section passes through the atrial appendages. The diminutive width of left atrium anterior to the right pulmonary veins is well shown. Søndergaard (1954) was the prime 


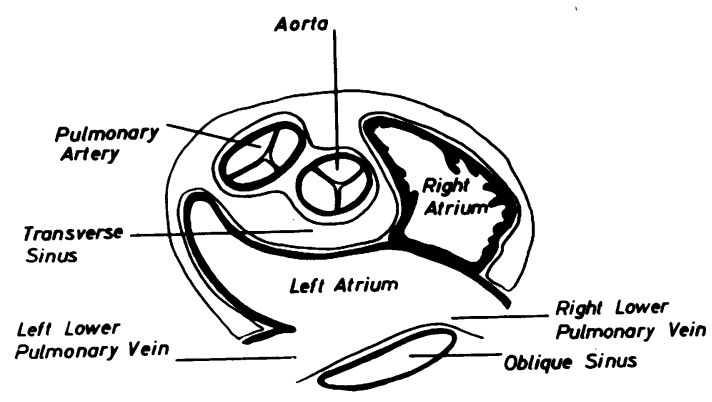

FIG. 1. Cross-section through the atria at the level of the appendages and transverse sinus, which serves to illustrate the relationship of the left anterior atrial wall to the transverse sinus, of the atrial wall between the venous orifices to the oblique sinus, and the relatively limited extent of atrial wall which lies anterior to the venous orifices, especially on the right.

exponent of development of the cleft between the left and right atria at this level for the purposes of circumferential passage of a constricting suture about an atrial septal defect for the purpose of closure of the defect without assistance from cardiopulmonary bypass. Cross-sections at more caudal levels (Fig. 2) serve to confirm that the normal left atrium is an almost exclusively posterior structure, and that, even on the left, there is a small extent of left atrial wall in front of the pulmonary veins.

In mitral stenosis left atrial enlargement is confidently recognized radiographically-by obtrusion on the left heart border of the enlarged left atrial appendage between the boss which represents the right ventricular outflow or pulmonary arterial segment, rostrally, and the left ventricular border, caudally, and by the density of the body of the enlarged left atrium behind the ventricles and the right atrium (Fig. 3). For this density to have a sharply defined margin, it must lie in contact with and be contrasted against aerated lung, or have an edge in which there is calcium, and a ca'cified atrial wall is excessively rare.

The left atrium can enlarge to the right, to the left, or both, in front of or behind the venous orifices; posteriorly and laterally, between the venous orifices, and caudal to them ; and also rostrally, both anteriorly, in the transverse sinus, and posteriorly, but in this direction obstruction to enlargement is more consistently encountered, in the form of the pulmonary arterial trunk and its right and left branches, and the tracheal bifurcation and the right and left main bronchi (see Fig. 11).

Illustrative radiographic evidence, supported by appropriate placement of cardiac catheters, and bronchography (Figs 4 to 10), substantiate these statements, and corroborative evidence has been

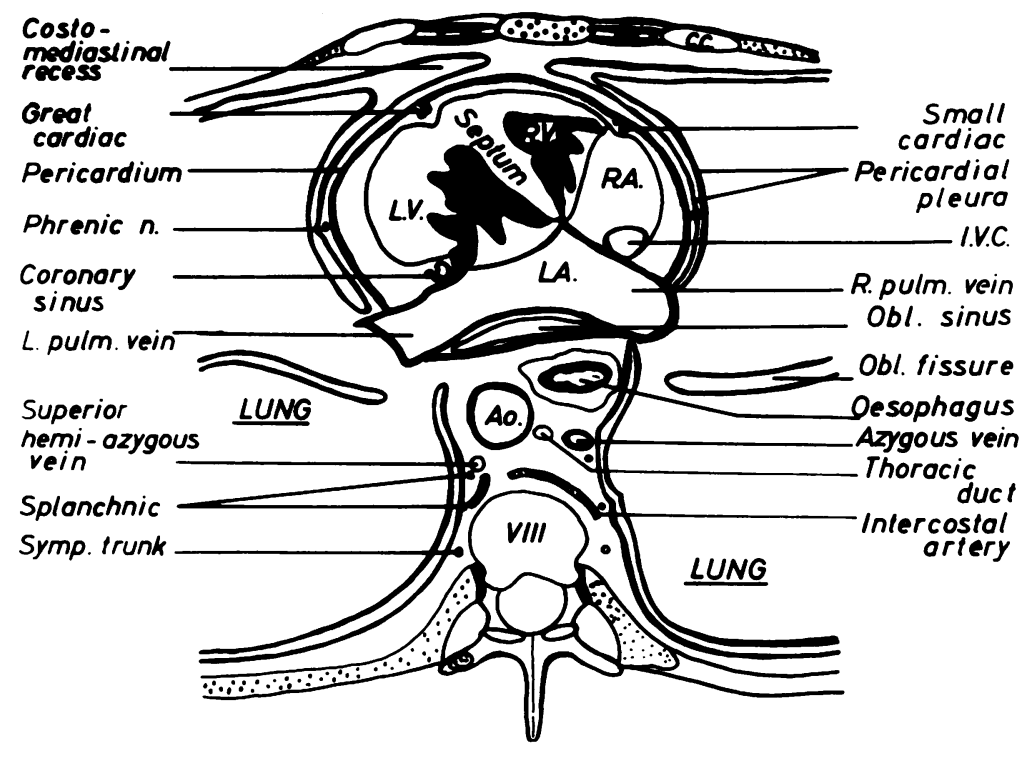

FIG. 2. Cross-section at the level of the 7th thoracic intervertebral disc, caudal to the level of the atrial appendages, which serves to demonstrate the posterior relations of the left atrium. 


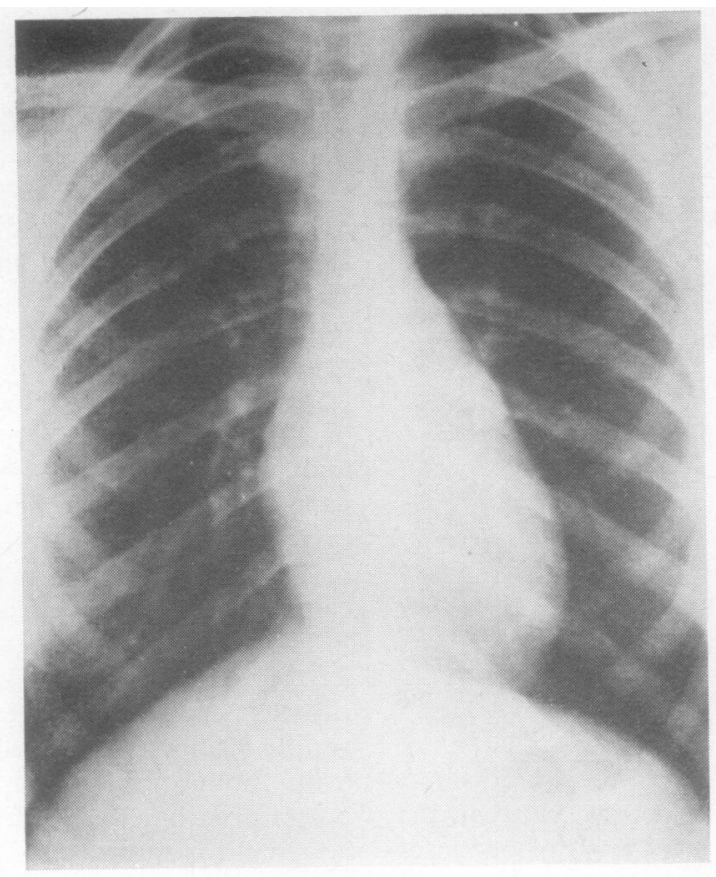

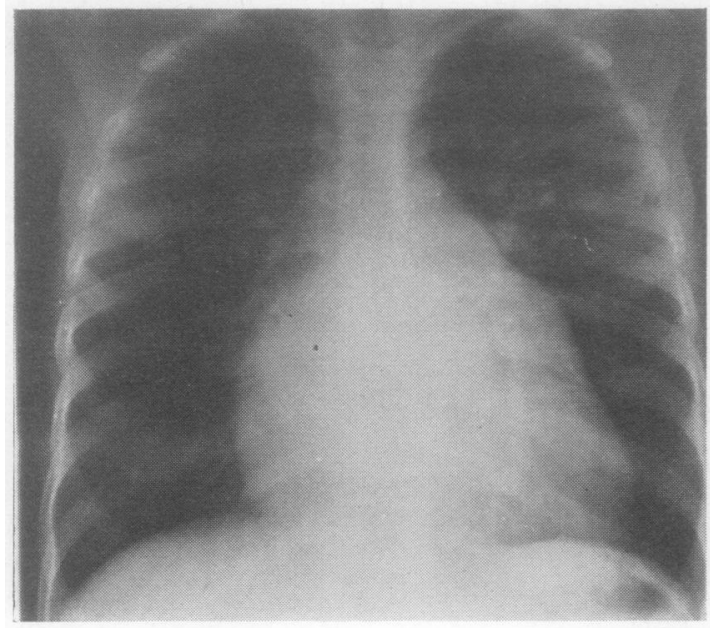

(b)

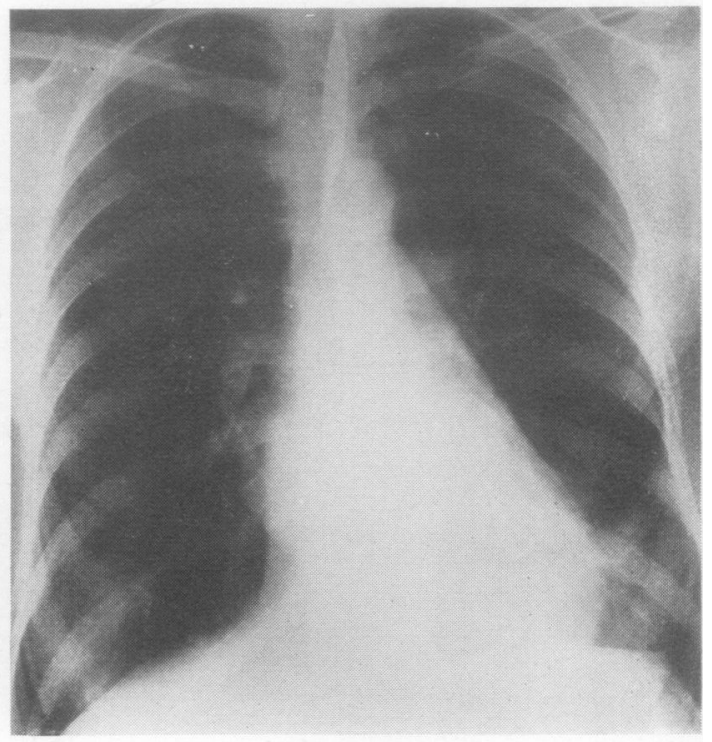

(c)

FIG. 3. Three examples of radiographic appearances unmistakably those of mitral stenosis. In (a), the heart is unusually slender but the left atrial appendage makes its accustomed appearance on the left heart border, and the right-sided double contour, the consequence of left atrial enlargement posteriorly, between the venous orifices is clear. In (b), the cardiac contour is essentially the same, although the heart is much larger and there is considerable right atrial enlargement. The double contour at the right heart border is again clear. In (c), previous mitral valvulotomy with amputation of the left atrial appendage has modified the left heart contour. The left atrium constitutes much of the right heart border, projects beyond the right atrium, and its greatest right-sided extension is behind the pulmonary hilum. 


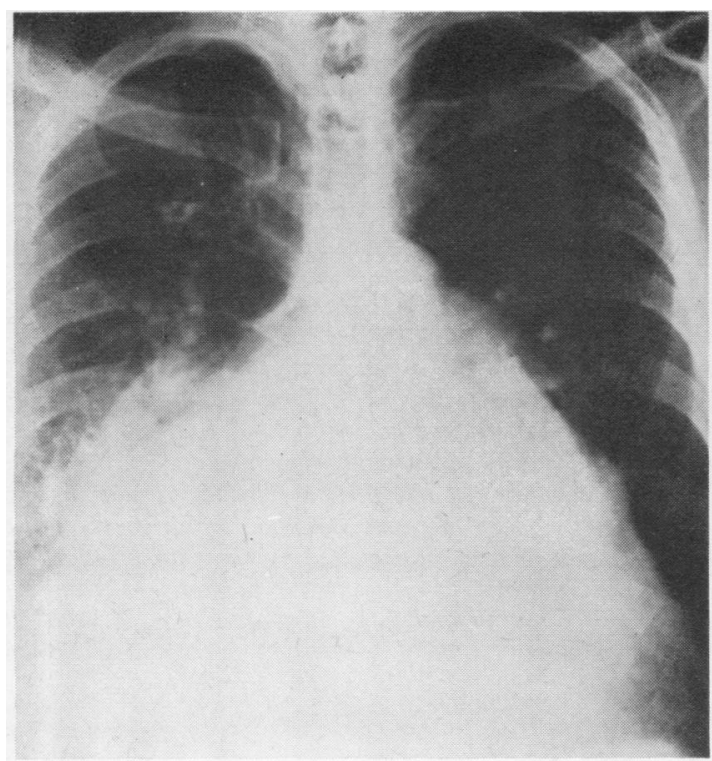

(a)

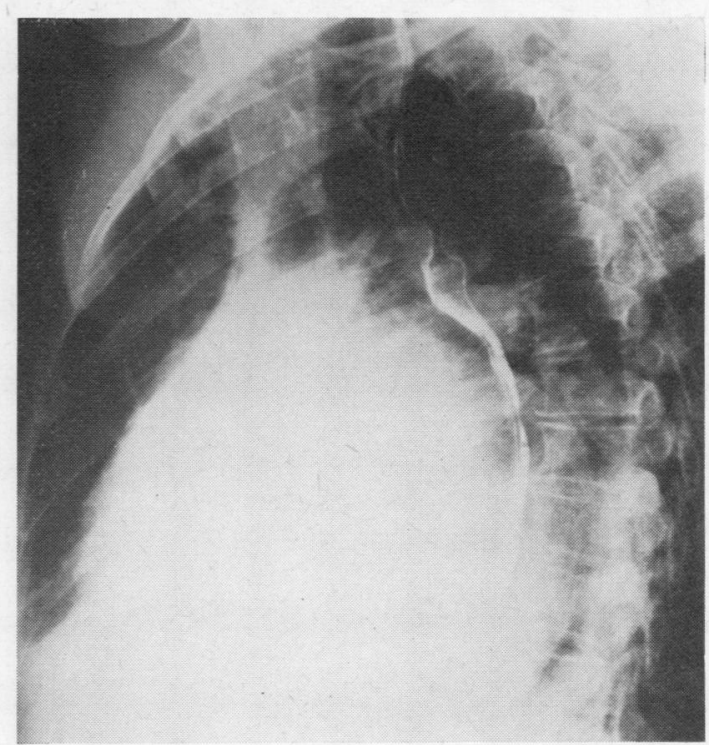

(b)

FIG. 4. (a) Postero-anterior and (b) left oblique views which show gross left atrial enlargement, mainly to the right, and the oesophagus displaced posteriorly, but also 'slipped' so that there is atrial enlargement posterior to the column of barium.

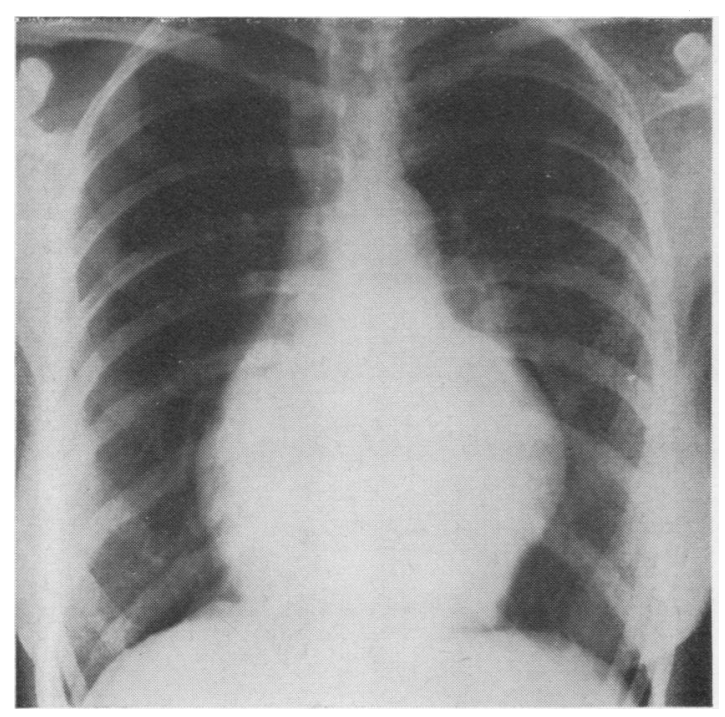

FIG. 5. The atrium is greatly enlarged, 'aneurysmal' to the left, and the enlargement is certainly not symmetrical. In this instance the greatest extent of left atrial enlargement seen at thoracotomy was anterior to the left pulmonary veins. The left atrial appendage was enormous but does not constitute any part of the cardiac silhouette in the postero-anterior view. There is visible a double contour where left atrium and left ventricle are superimposed and contrasted against aerated lung medially in the 10th left posterior intercostal space.

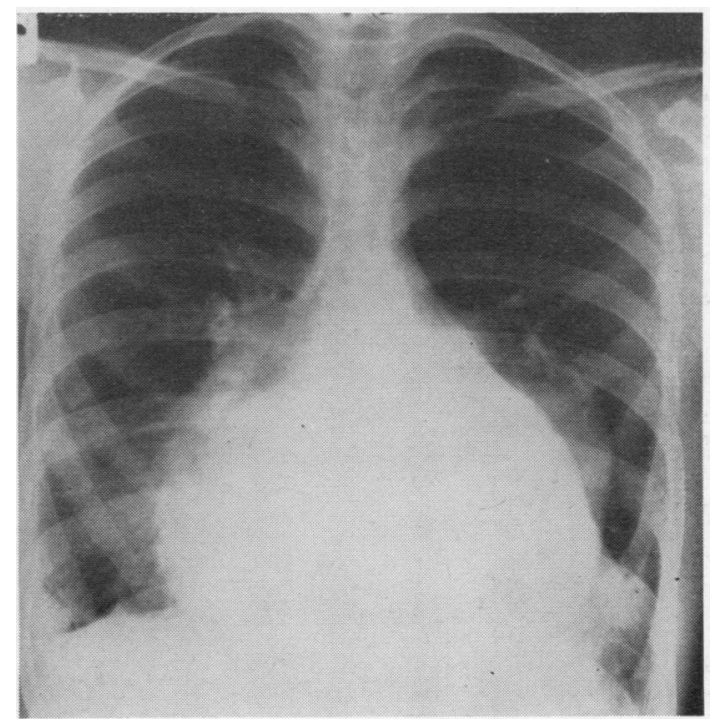

FIG. 6. Postero-anterior chest radiograph in which is shown massive left atrial enlargement to the right and to the left. Both heart borders are left atrium and there are bilateral double contours, on the right the consequence of left atrial enlargement, behind the pulmonary hilum, with right atrial edge superimposed in front of the hilum; on the left, from left atrium posterior to the pulmonary hilum, rostrally and caudally, superimposed on left atrium and grossly enlarged left atrial appendage in front of the pulmonary venous orifices. 


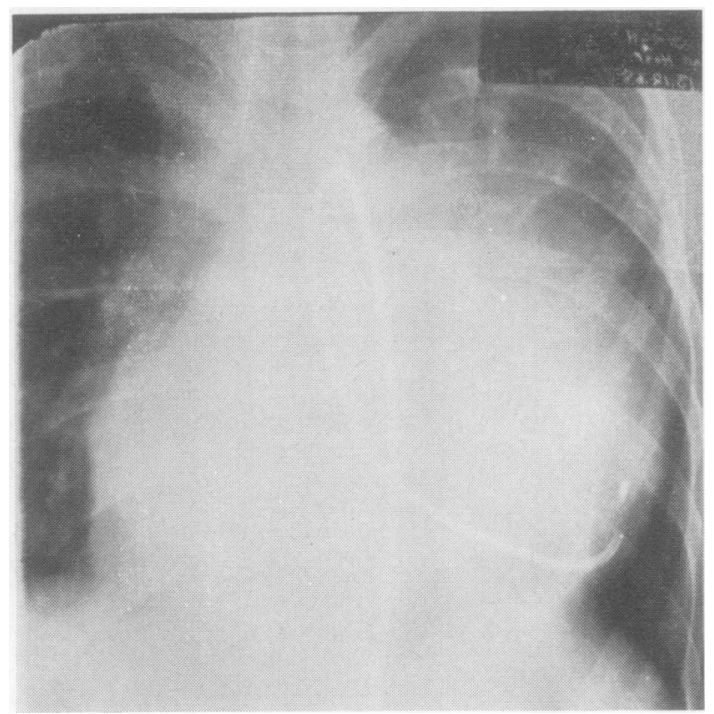

FIG. 7. A trans-aortic left atrial catheter demonstrates the left atrial edge at the left heart border. At left thoracotomy, for mitral valve replacement in this instance, left atrial enlargement was both in front and behind the left $\vec{w}$ pulmonary veins, predominantly behind, with a massively enlarged left atrial appendage. The double contour here of left atrium against left atrium is clear; a cleft in the N atrial wall is occupied by the lingular segment; the most on lateral extent of atrium, occupied by the catheter tip, lies posteriorly, and bulges laterally the pulmonary ligament; the dense, more medial edge lies anteriorly, caudal to the left pulmonary vein.

FIG. 7
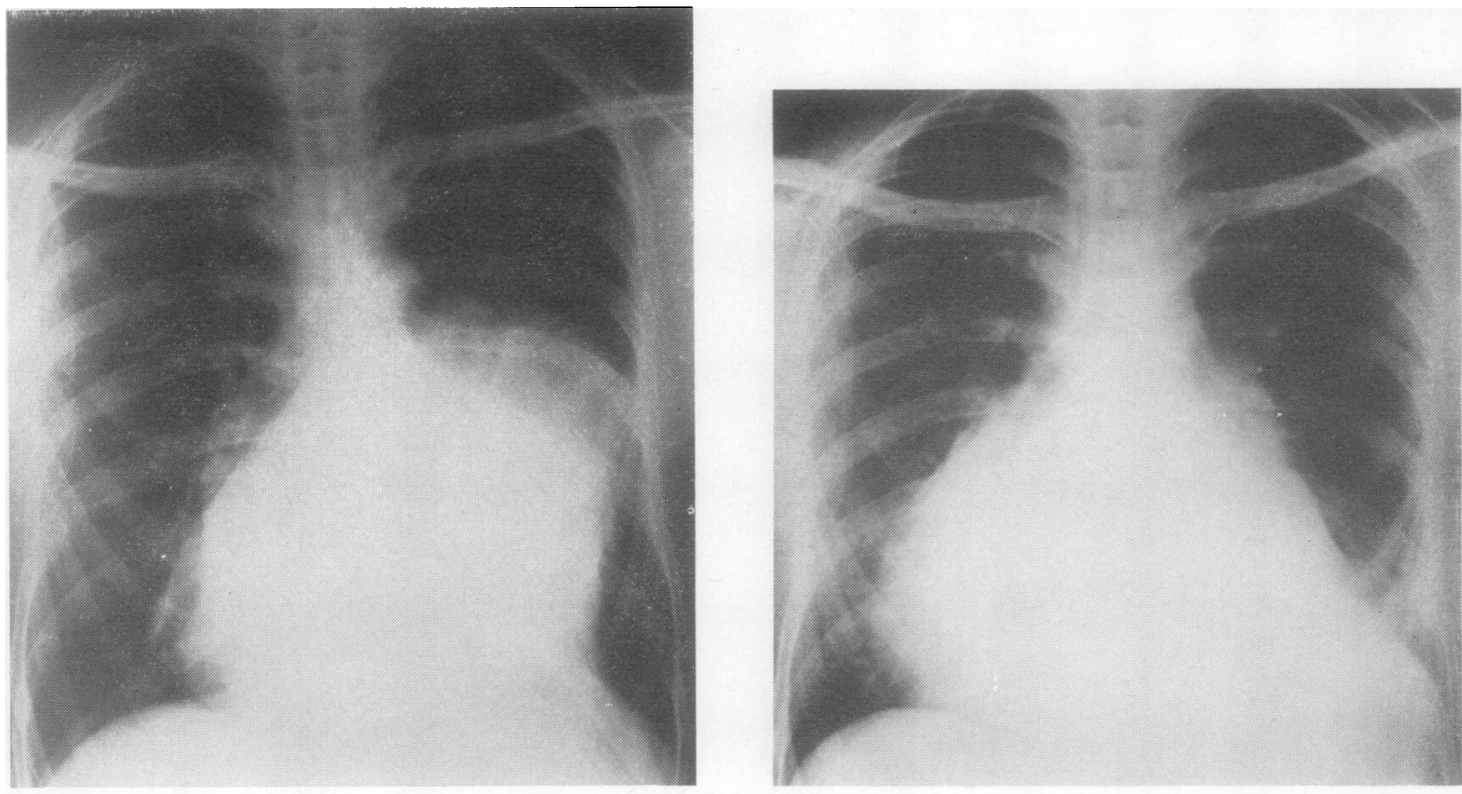

(a)

(19. to the left, but also to the right, with bilateral double contours. At left thoracotomy for mitral valve replacement $\square$ the left atrial enlargement was almost exclusively anterior to the left pulmonary veins, which were displaced far posteriorly, and the left atrial appendage was also massively enlarged. The relationship of the right-sided extension of $\stackrel{\mathbb{\Phi}}{\Omega}$ the left atrium to the right pulmonary venous orifices was assessed from inspection and palpation of the interior of the left $\overrightarrow{\mathbb{D}}$ atrium, and was exclusively posterior to the right venous orifices. (b) Post-operative postero-anterior chest radio- 0 graph after mitral valve replacement and left atrial trimming, which was confined to that area of atrium in front of, rostral and caudal to the left pulmonary vein. 


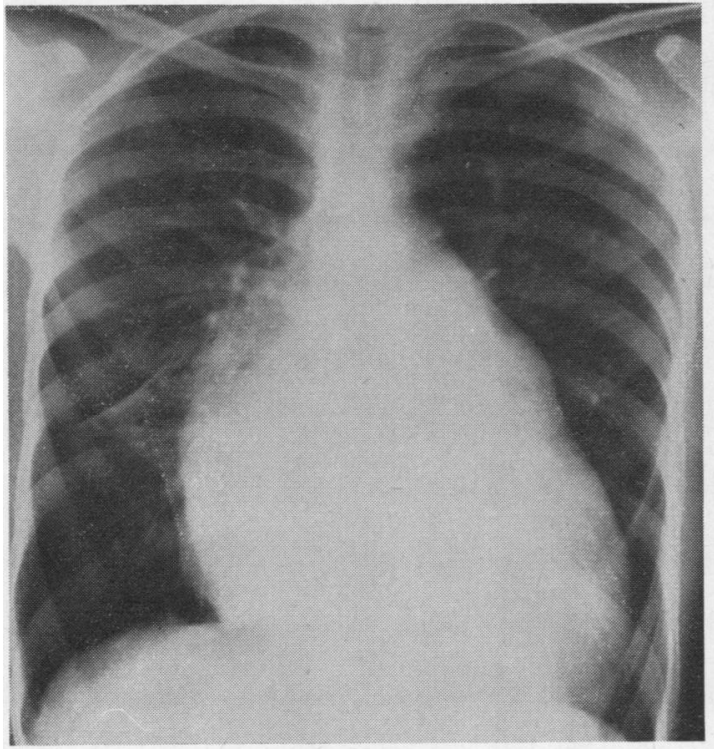

(a)

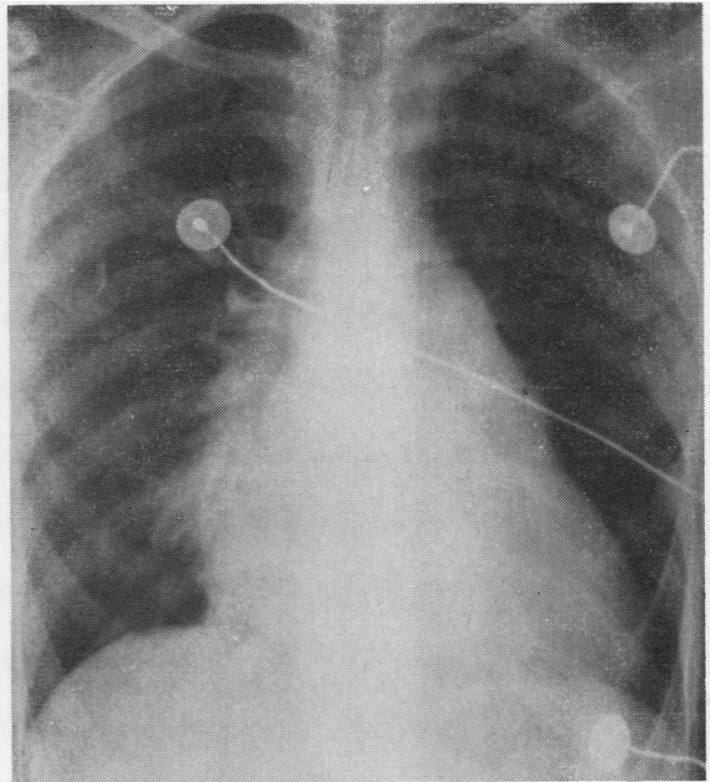

(b)

FIG. 9. (a) Postero-anterior chest radiograph which shows left atrial enlargement, predominantly to the right, with double contours; the left atrium forms nearly the whole of the right heart border. The caudal projection was seen at right thoracotomy for mitral valve replacement to lie posterior to the level of the venous orifices and to displace laterally the right pulmonary ligament. The rostral projection displaced forward the superior vena cava and extended to the right in front of the right pulmonary vein. The triangular pulmonary opacity based on the right heart border was the medial segment of the middle lobe, which was shrunken and partially airless. (b) Antero-posterior chest radiograph made on the day following mitral valve replacement and paring of the left atrium through a right thoracotomy. The broad ellipse of left atrium excised almost encircled the right pulmonary veins, the orifices of which were separated by $11 \mathrm{~cm}$. from those of the left pulmonary vein.

FIG. $10 \mathrm{a}$

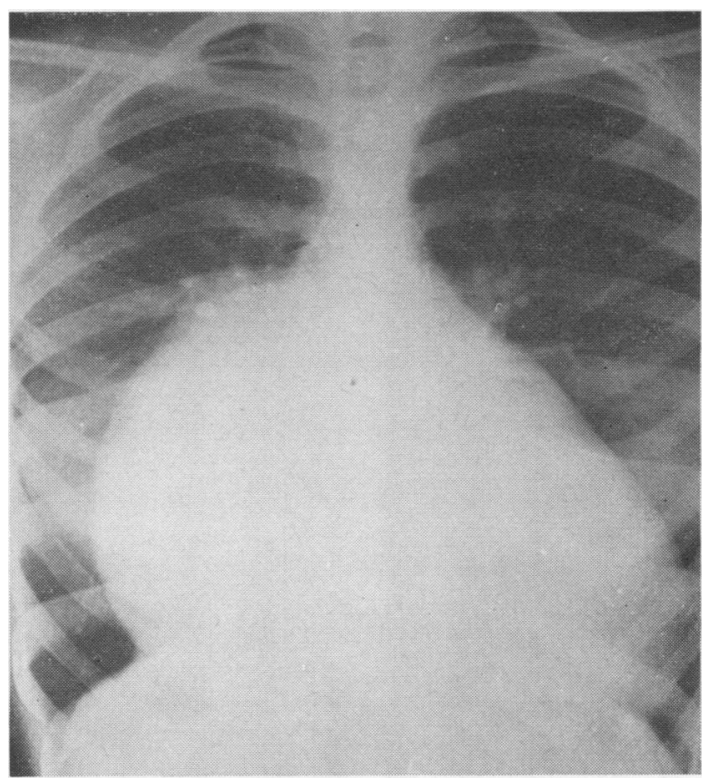

Legend to Fig. 10a is on page 197, overleaf. 


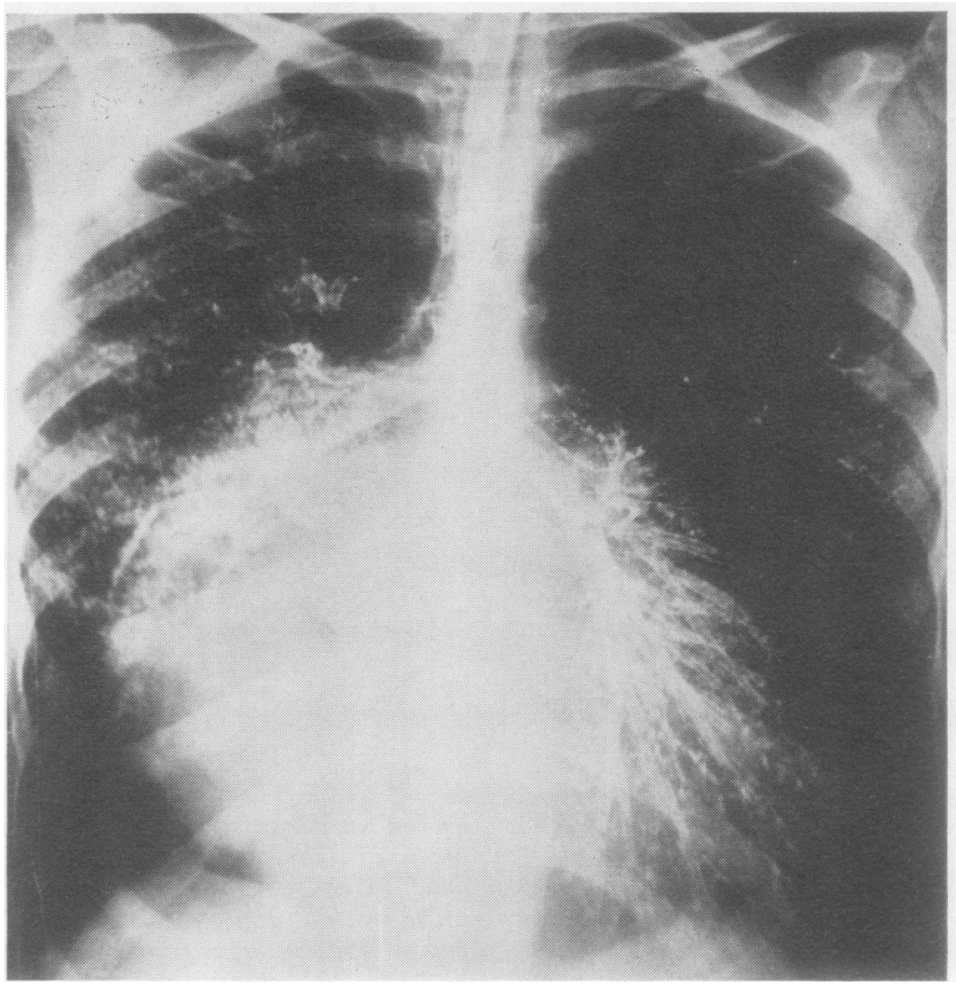

FIG. $10 \mathrm{c}$

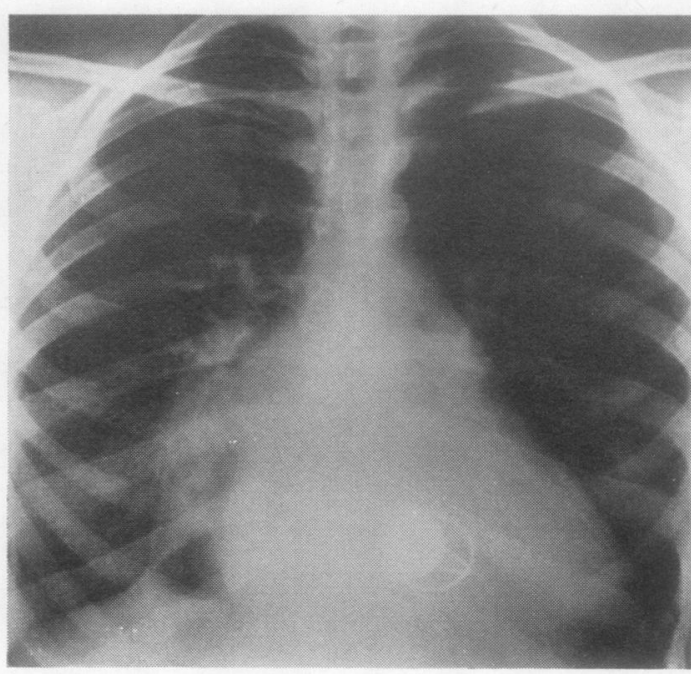

FIG. 10d

Legend to Figs 10b, 10c, and 10d is on facing page. 


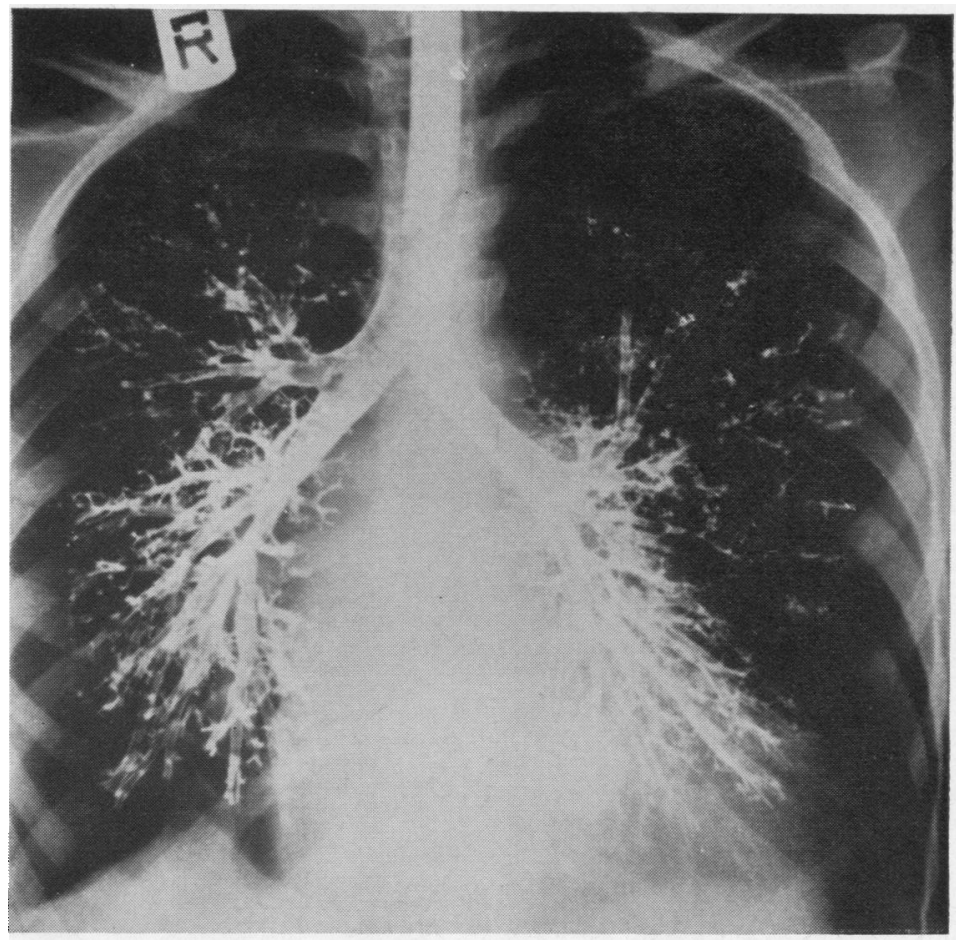

FIG. $10 \mathrm{e}$

FIG. 10. These radiographs are of the same patient; (a) a postero-anterior chest radiograph in which is shown enlargement of the heart predominantly right-sided in a patient with pure mitral incompetence and a giant left atrium. The antero-posterior bronchogram (b) shows the main bronchi splayed. (c) Two cardiac catheters have been passed, one transvenously from the arm into the right atrium, and here seen against the right lateral wall of the right atrium, before the catheter turns upwards into the right ventricle and pulmonary artery, with the catheter well medial to the right heart border; the other, passed from the groin, up the aorta, through the left ventricle into the left atrium and right upper lobar pulmonary vein, and here seen to lie against the right heart border, thereby established as left atrium. (d) A postero-anterior chest radiograph made two weeks after mitral valve replacement with a Starr-Edwards prosthesis. The heart size is greatly diminished by amputation of a large ellipse of left atrium, the incision extending from in front of the right pulmonary veins, caudally, and then medially and rostrally between the right and left orifices, which, in this instance, were separated by a distance of $12 \mathrm{~cm}$. $A$ post-operative antero-posterior bronchogram (e) shows that the main bronchi are no longer splayed.

obtained angiographically and with the heart exposed at thoracotomy. Right- or left-sided, or bilateral, double contours at the heart border are common radiographic features of giant atrium. For there to be double contours there must be bosses on the cardiac surface, with folds which distinguish the bulges, each contrasted against aerated lung. Aerated lung must be interposed in a cleft in the atrial wall, usually in relation to the atrial septum or the entrance of the pulmonary veins, the orifices of which are seldom themselves greatly widened; or the opportunity for the demonstration of radiographic contrast may occur where atrium and ventricle are superimposed, with lung between. The atrium enlarges asymmetrically and its surface is thrown into folds by 


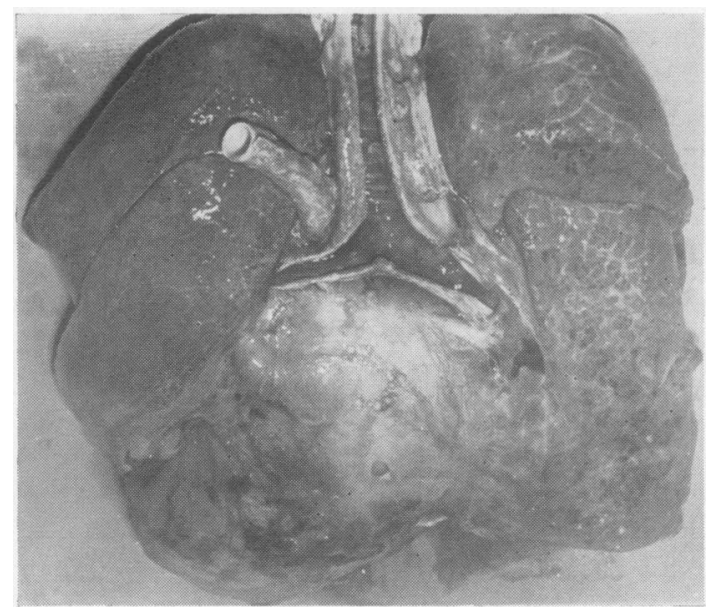

FIG. 11. Post-mortem specimen in which can be seen splaying of the main bronchi so gross that their direction is almost transverse. The transected aorta is artificially displaced upwards to facilitate vision. The left lower bronchus was, in this instance, totally obstructed by external compression. The massive dilatation of the left atrium is mainly between the right and left venous orifices and extends far caudally, and rostrally as far as adjacent structures permit. venous attachments and where enlargement is dis couraged by contact with structures relativeliz resistant to displacement. When folds do not de응 velop on the surface of the giant atrium and lung does not occupy a cleft in the atrial wall, the op@ portunity for radiographic contrast is lost and iफ्फि these circumstances a double contour is not seen-

The effect of surgical reduction in size of a. giant atrium can be judged radiographically (Fig $\overrightarrow{\delta . ~}$ 8 to 10) and bronchographically (Fig. 10); its value can only be assumed. In none of the speci户 mens of resected atrium was there evidence of atrial infarction.

\section{REFERENCES}

Daley, R., and Franks, R. (1949). Massive dilatation of the left auricle? Quart. J. Med., 18, 81 .

Kent, E. M., Fisher, D. L., Ford, W. B., and Neville, J. F. (1956) Mitral valve surgery and left heart catherization in giant lefo atrium. Arch. Surg., 73, 503.

Le Roux, B. T., and Williams, M. A. (1969). Dysphagia megala $\frac{\widehat{\Omega}}{-}$ triensis. Thorax, 24, 603

Owen, I., and Fenton, W. J. (1901). A case of extreme dilatation of the left auricle of the heart. Trans. clin. Soc. Lond., 34, 183.

Parsonnet, A. E., Bernstein, A., and Martland, H. S. (1946). Massive left auricle with special reference to its etiology and mechanism Amer. Heart J., 31, 438.

Søndergaard, T. (1954). Closure of atrial septal defects. Acta chir scand., 107, 492.

Venner, A. (1954). Massive left atrium and mitral valvotomy. Brißn med. J., 1, 1359. 